TITLE:

\title{
Effect of Spatial Homogeneity of Spin Polarization on Magnetic Field Response of an Optically Pumped Atomic Magnetometer Using a Hybrid Cell of K and Rb Atoms
}

\section{AUTHOR(S):}

Ito, Yosuke; Ohnishi, Hiroyuki; Kamada, Keigo; Kobayashi, Tetsuo

\section{CITATION:}

Ito, Yosuke ... [et al]. Effect of Spatial Homogeneity of Spin Polarization on Magnetic Field Response of an Optically Pumped Ato mic Magnetometer Using a Hybrid Cell of K and Rb Atoms. IEEE Transactions on Magnetics 2012, 48(11): 3715-3718

\section{ISSUE DATE:}

2012-11

URL:

http://hdl.handle.net/2433/161658

\section{RIGHT:}

(c) 2012 IEEE. Personal use of this material is permitted. Permission from IEEE must be obtained for all other uses, in any current or future media, including reprinting/republishing this material for advertising or promotional purposes,

creating new collective works, for resale or redistribution to servers or lists, or reuse of any copyrighted component of this work in other works.; この論文は出版社版でありません。引用の際には出版社版をご確認ご利用ください。; This is not the published version. Please cite only the published version. 


\title{
Effect of Spatial Homogeneity of Spin Polarization on Magnetic Field Response of an Optically Pumped Atomic Magnetometer Using a Hybrid Cell of $K$ and Rb Atoms
}

\author{
Yosuke Ito $^{1,2}$, Hiroyuki Ohnishi ${ }^{1}$, Keigo Kamada $^{1}$, and Tetsuo Kobayashi ${ }^{1}$ \\ ${ }^{1}$ Graduate School of Engineering, Kyoto University, Kyoto, Kyoto, Japan \\ ${ }^{2}$ Advanced Biomedical Engineering Research Unit, Kyoto University, Kyoto, Kyoto, Japan
}

\begin{abstract}
We measured the spatial homogeneity of spin polarization, which is directly related to the sensitivity and magnetic linewidth of an optically pumped atomic magnetometer with a hybrid cell of $K$ and $R b$ atoms. This was done by changing the position of the probe beam. Furthermore, for comparison, we also measured the sensitivity and magnetic linewidth of atomic magnetometers with single $K$ and single $\mathbf{R b}$ cells, and found that optically thick atoms were spin polarized homogeneously with the hybrid cell. Optically thin alkali metal vapor can be spin polarized homogeneously, and the spin polarization transfers to an optically thick alkali metal vapor. An atomic magnetometer with the hybrid cell was found to be more effective in realizing simultaneous signal measurements at different locations with the uniform sensitivity and magnetic linewidth.
\end{abstract}

Index Terms - Atomic magnetometer, Biomagnetics, Optical pumping, Spin exchange.

\section{INTRODUCTION}

$\mathrm{O}$ PTICALLY pumped atomic magnetometers have gained considerable attention in recent years because of their potential application to biomagnetic measurements. Unlike superconducting quantum interference devices (SQUIDs), atomic magnetometers have an advantage in that they do not require cryogenic cooling. Furthermore, the sensitivity of atomic magnetometers has been theoretically estimated to be $0.01 \mathrm{fT} / \mathrm{Hz}^{1 / 2}$ under spin exchange relaxation free (SERF) conditions [1]-[3]. We have also studied the potential application of the magnetometer to biomagnetic measurements by performing phantom measurements [4], [5].

Well-known atomic magnetometers use single alkali metals in a glass cell. In this case, it is difficult to deal with optically dense/thick alkali metal vapors because of the light attenuation of the pump beam through the cell. Here, an optically thick/dense vapor is one whose optical depth is much larger than 1 and the optical depth for photons of frequency $v$ is given by

$$
\mathrm{OD}=n \sigma(v) l
$$

where $n$ is the density of the vapor, $\sigma(v)$ is the absorption cross section, and $l$ is the length of the light path.

In contrast, we have developed a magnetometer that uses a hybrid cell containing optically thin $\mathrm{K}$ and dense $\mathrm{Rb}$ vapors [6]. By using a hybrid magnetometer, Romalis has also demonstrated a spin polarization that is 4.5 times higher than that obtained by direct optical pumping of only $\mathrm{K}$ [7]. The dense atoms can be spin polarized via spin-exchange collisions with other types of thin spin-polarized atoms. This method is expected to achieve homogeneous spin polarization throughout the cell. Homogeneous spin polarization is

Manuscript received January 1, 2012 (date on which paper was submitted for review). Corresponding author: Y. Ito (e-mail: yito@kuee.kyoto-u.ac.jp).

Digital Object Identifier inserted by IEEE advantageous mainly in imaging modalities such as magnetocardiography (MCG), magnetoencephalography (MEG), and magnetic resonance imaging (MRI). However, there have been no experimental demonstrations to show this homogeneity of spin polarization with hybrid optical pumping.

In this study, we carried out sensitivity measurements to assess the spatial homogeneity of spin polarization in a K-Rb hybrid cell to verify its effect on the magnetic field response. Furthermore, for comparison purposes, we also investigated the spatial homogeneity of spin polarization of single $K$ and single $\mathrm{Rb}$ cells.

\section{METHODS}

Fig. 1 illustrates the experimental setup in this study. The sensor head of the magnetometer used in this study was a cubic Pyrex glass cell whose size was $3 \mathrm{~cm} \times 3 \mathrm{~cm} \times 3 \mathrm{~cm}$, and within which helium and nitrogen were enclosed as buffer gases at a ratio of $10: 1$, and at a total pressure of $150 \mathrm{kPa}$ at room temperature. By increasing the temperature to $453 \mathrm{~K}$, the densities of gaseous $\mathrm{K}$ and $\mathrm{Rb}$ in the hybrid cell were $1 \times 10^{13}$ $\mathrm{cm}^{-3}$ and $2 \times 10^{14} \mathrm{~cm}^{-3}$, respectively. In addition, we prepared a single $\mathrm{K}$ cell and a single $\mathrm{Rb}$ cell, which were under conditions similar to those of the hybrid cell, except that they contained only one type of alkali metal atoms. The glass cell was set in a magnetic shield whose shielding factor is greater than $10^{6}$ at $100 \mathrm{~Hz}$ and a three-axis field coil system.

We used a Ti-Sapphire laser as a pump laser, an externalcavity diode laser to probe $\mathrm{Rb}$ atoms, and a distributed feedback (DFB) laser to probe $\mathrm{K}$ atoms. The wavelength of the pump beam was tuned to the D1 resonant wavelength of the alkali metal atoms. The wavelength of the probe beam was slightly detuned from their D1 resonant wavelength, i.e., $770.00 \mathrm{~nm}$ for $\mathrm{K}$ atoms and $794.38 \mathrm{~nm}$ for $\mathrm{Rb}$ atoms. To ensure uniform exposure over the entire cell, we sufficiently expanded the pump beam with beam expander optics and shaped its profile into a square $3 \mathrm{~cm}$ on a side with a square aperture of $3 \mathrm{~cm} \times 3 \mathrm{~cm}$, as illustrated in Fig. 1. Meanwhile, 


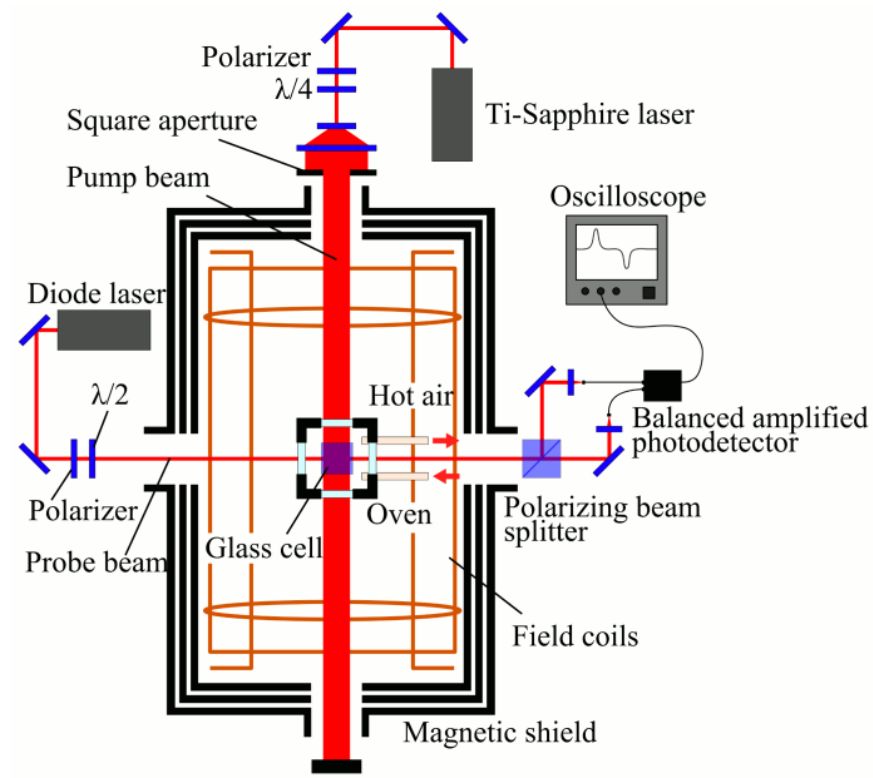

Fig. 1. Schematic of the experimental setup. The glass cell was placed near the center of the magnetic shields and heated to $453 \mathrm{~K}$ by hot air.

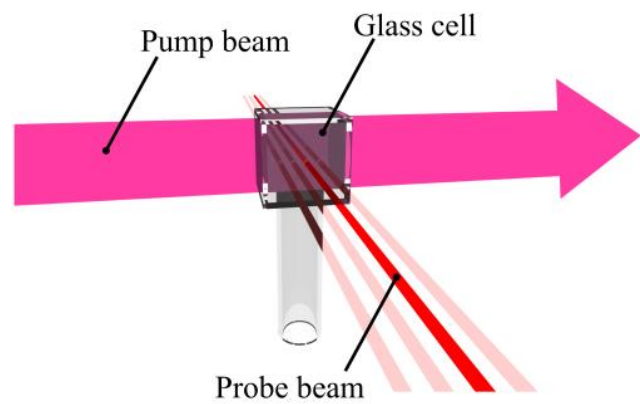

Fig. 2. Probe beam position. The probe beam is transmitted to four different locations, which are $5,10,15$, and $20 \mathrm{~mm}$ away from the pump beam inlet face of the glass cell.

the diameter of the probe beam was $4 \mathrm{~mm}$ and the power intensity was $1.5 \mathrm{~mW}$.

In the case of the hybrid cell, the $\mathrm{K}$ atoms were spin polarized by a circularly polarized pump beam, and the spin polarization was transferred to the $\mathrm{Rb}$ atoms by spin-exchange collisions. The spin polarization rotated around the external magnetic field that was orthogonal to both the pump and probe beams. Here, the polarization plane of a linearly polarized probe beam penetrating the group of spin-polarized atoms is rotated by the magneto-optical effect. The rotation angle of the plane corresponding to the magnetic field was obtained by measuring the difference between the intensities of the mutually perpendicular polarization components of the probe beam.

The resonant frequency of the atomic magnetometer was tuned to $100 \mathrm{~Hz}$ by a bias magnetic field that was parallel to the pump beam. This was done in order to circumvent the effect of the inevitable low-frequency environmental magnetic

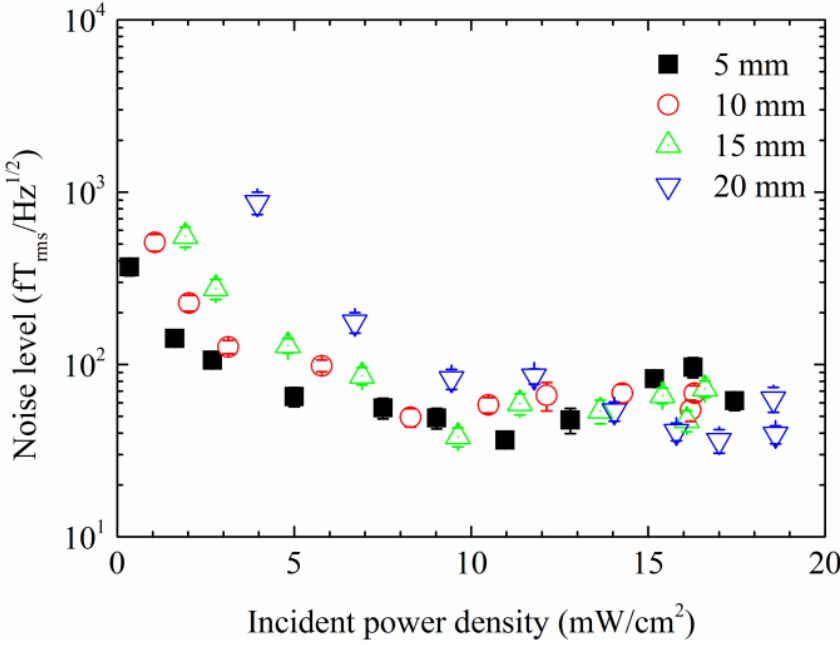

Fig. 3. Noise level at four different locations, which are 5, 10, 15, and 20 $\mathrm{mm}$ away from the pump-beam inlet face of the hybrid cell plotted against the incident pump beam power density.

noise. To estimate its noise level and magnetic linewidth, which refers to the spin relaxation rate of the polarized atoms, we applied a $100-\mathrm{Hz}$ magnetic field whose amplitude was 34 $\mathrm{pT}_{\mathrm{rms}}$ and 20 magnetic field signals ranging from 10 to $200 \mathrm{~Hz}$. We transmitted the probe beam at four different locations that were $5,10,15$, and $20 \mathrm{~mm}$ away from the pump-beam inlet face of the glass cell, as shown in Fig. 2.

\section{RESUlTS AND DISCUSSION}

Fig. 3 shows a plot of the noise level at four different locations of the hybrid cell against the incident pump beam power density. Below $10 \mathrm{~mW} / \mathrm{cm}^{2}$, the noise level increased with an increase in the distance from the pump-beam inlet face of the cell, whereas it was nearly saturated from 10 to 20 $\mathrm{mW} / \mathrm{cm}^{2}$. This is because the low incident pump beam power density causes insufficient spin polarization over the entire cell, whereas the high incident pump beam power density gives rise to sufficient spin polarization. This indicates that the spin polarization of $\mathrm{Rb}$ atoms at all the points was similar when a sufficient pump beam power density was induced. The highest sensitivity was approximately $30 \mathrm{fT}_{\mathrm{rms}} / \mathrm{Hz}^{1 / 2}$.

Fig. 4 provides plots of the magnetic linewidth at four different locations of the hybrid cell against the incident pump beam power density. The linewidth increased with an increase in the pump beam power. The farther away from the pumpbeam inlet face the probe beam transmits, the larger is the magnetic linewidth. These results are caused by the fluctuation of the spin polarization caused by deexcited lights from optically pumped $\mathrm{K}$ atoms. Therefore, at the points far away from the pump-beam inlet face, the effect of the pump beam was smaller. Moreover, at approximately $0 \mathrm{~mW} / \mathrm{cm}^{2}$, a spin relaxation of approximately $5 \mathrm{~Hz}$ was caused by spindestruction collisions and the probe beam. 


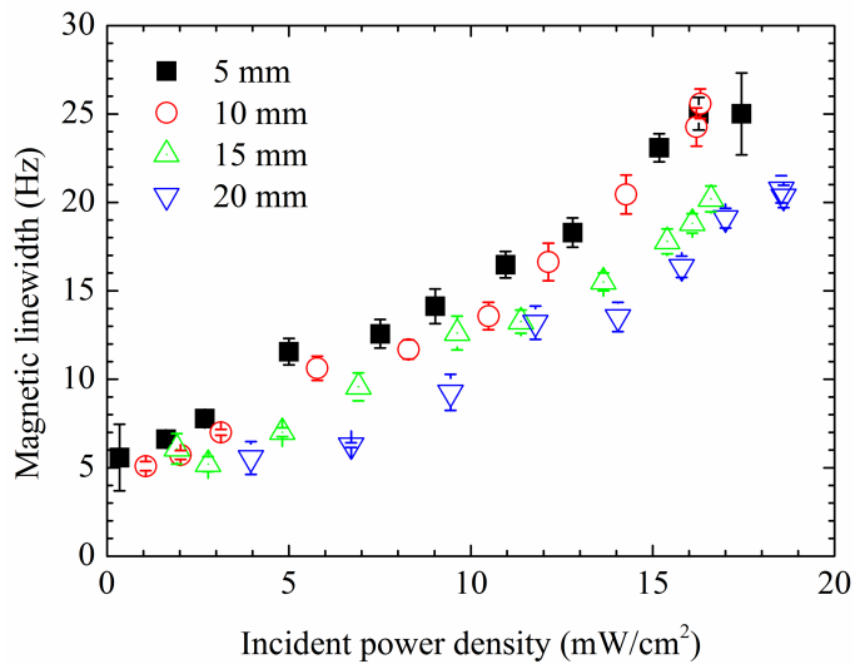

Fig. 4. Magnetic linewidth at four different locations, which are 5, 10, 15, and $20 \mathrm{~mm}$ away from the pump-beam inlet face of the hybrid cell plotted against the incident pump beam power density.

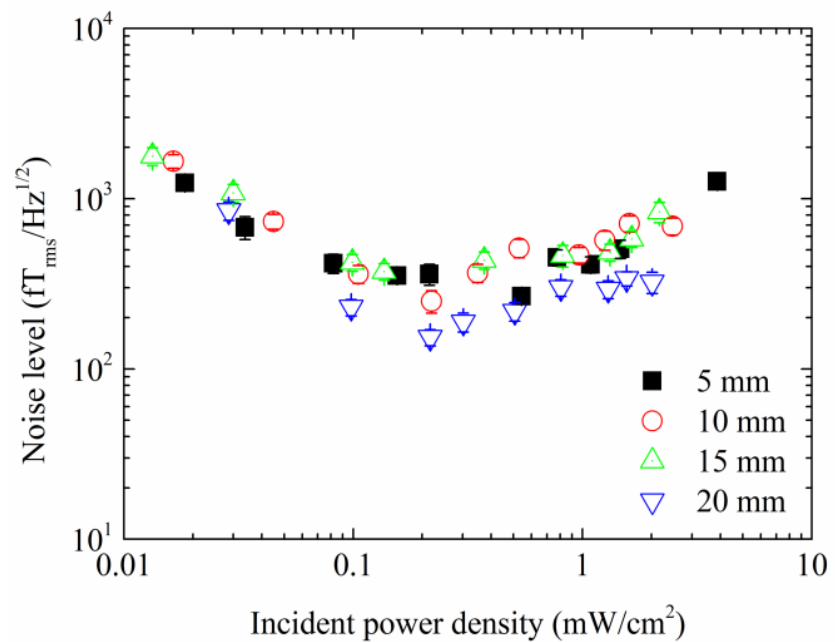

Fig. 5. Noise level at four different locations, which are 5, 10, 15, and 20 $\mathrm{mm}$ away from the pump-beam inlet face of the $\mathrm{K}$ cell plotted against the incident pump beam power density.

In Fig. 5, the noise level at four different locations of the $\mathrm{K}$ cell is plotted against the incident pump beam power density. In the case of the $\mathrm{K}$ cell, the highest sensitivity was observed at approximately $0.2 \mathrm{~mW} / \mathrm{cm}^{2}$. This value is much smaller than that of the hybrid cell because of the smaller density of probed atoms compared with the hybrid cell. In this case, a much lower pump beam power density was therefore sufficient to obtain optimal sensitivity. Fig. 6 shows the magnetic linewidth at four different locations of the $\mathrm{K}$ cell plotted against the incident pump beam power density. The linewidth shows a sharp rise since it was dominated by the spin relaxation by the pump beam. At several milliwatts per centimeter square, the magnetic linewidth was several hundred hertz. The tendency and value were similar for all the measured points. That is, the sensitivity and magnetic linewidth were more significantly affected by the pump beam power density than those of the hybrid cell. The thin alkali metal vapor can be spin polarized homogeneously.

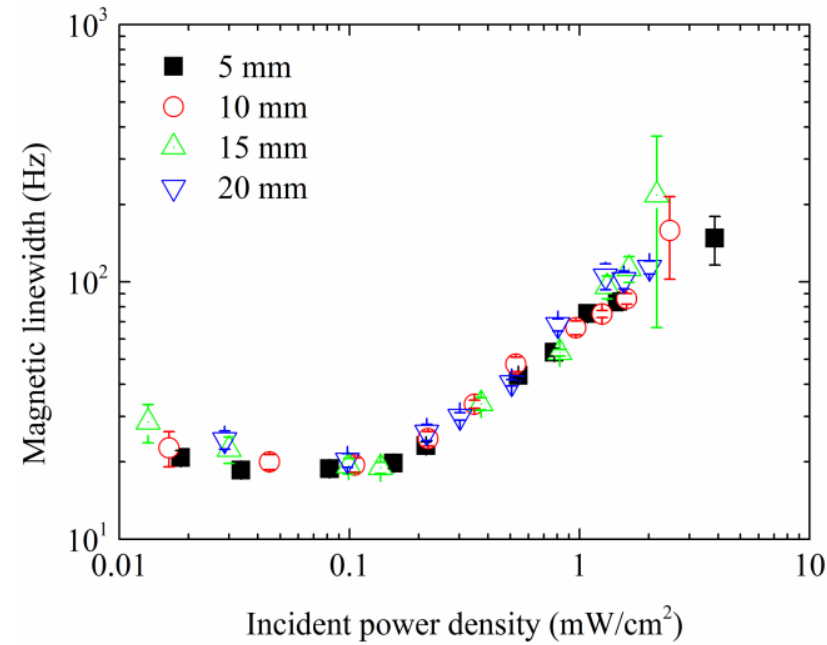

Fig. 6. Magnetic linewidth at four different locations, which are 5, 10, 15, and $20 \mathrm{~mm}$ away from the pump-beam inlet face of the $\mathrm{K}$ cell plotted against the incident pump beam power density.

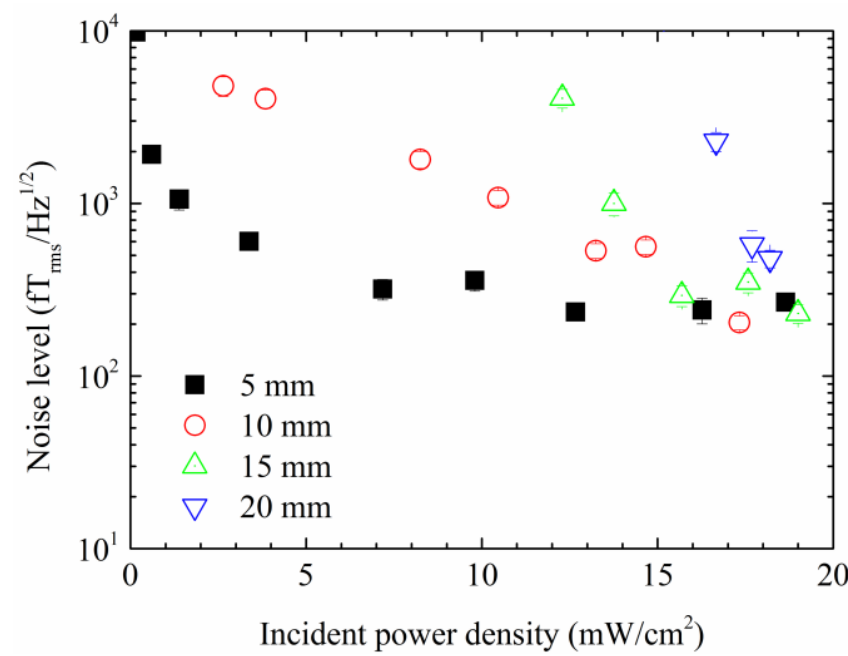

Fig. 7. Noise level at four different locations, which are 5, 10, 15, and 20 $\mathrm{mm}$ away from the pump-beam inlet face of the Rb cell plotted against the incident pump beam power density.

Finally, Fig. 7 shows the noise level at four different locations of the $\mathrm{Rb}$ cell as a function of the incident pump beam power density. Because of its high $\mathrm{Rb}$ density, the dependence of the noise level on the distance from the pumpbeam inlet face of the cell is clearly observed. At the points that were 15 and $20 \mathrm{~mm}$ away, we could not measure the noise level below incident pump beam power densities of 12 and 16 $\mathrm{mW} / \mathrm{cm}^{2}$, respectively. This implies that the noise level was higher than the test signal of $34 \mathrm{pT}_{\mathrm{rms}} / \mathrm{Hz}^{1 / 2}$. The noise level at all points reached a similar level when the incident pump beam power density was larger than at least $18 \mathrm{~mW} / \mathrm{cm}^{2}$. By comparing the $\mathrm{Rb}$ cell to the hybrid cell, we can realize homogeneous spin polarization over the entire cell with a lower pump beam power density.

In this study, we applied three types of glass cells, i.e., a hybrid cell, a $\mathrm{K}$ cell, and a $\mathrm{Rb}$ cell, to an optically pumped atomic magnetometer. Although they showed different characteristics, we consider that the difference was caused by 
the difference of the density of the alkali metal atoms, and not by the difference of the species. In the case of a single alkali metal cell, such as the $\mathrm{K}$ cell and $\mathrm{Rb}$ cell, dense alkali metal atoms $\left(10^{14} \mathrm{~cm}^{-3}\right.$ order $)$, which have the advantage of high signal intensity, cannot easily obtain sufficient and homogeneous spin polarization over the entire cell although smaller alkali metal density $\left(10^{13} \mathrm{~cm}^{-3}\right.$ order $)$ can be easily optically pumped over the entire cell.

In contrast, in the case of the hybrid cell, we can obtain a greater degree of homogeneous spin polarization when compared with the single alkali metal cell with dense alkali metal atoms. This is because we can polarize optically thin alkali metal atoms, after which the spin polarization transfers to the optically thick atoms. Therefore, by using atomic magnetometers with the hybrid cell, we are able to realize simultaneous signal measurements at different locations with the uniform sensitivity and magnetic linewidth, such as 3-D measurements of magnetic fields reported by Gusarov et al. [8].

\section{CONCLUSION}

We have shown the spatial homogeneity of spin polarization of a hybrid cell by measuring its sensitivity and magnetic linewidth by making comparisons with a $\mathrm{K}$ cell and a $\mathrm{Rb}$ cell. Based on the results for the $\mathrm{K}$ cell, the thin alkali metal vapor can be spin polarized over the entire cell. We found that the K-Rb hybrid cell can realize a greater degree of homogeneous spin polarization than the $\mathrm{Rb}$ cell because the spin polarization of the thin $\mathrm{K}$ atoms transfers to the dense $\mathrm{Rb}$ atoms over the entire cell because of spin-exchange collisions. In future studies, we plan to measure MCG and/or MEG distributions by using an optically pumped atomic magnetometer with the K-Rb hybrid cell.

\section{ACKNOWLEDGMENT}

This work was partly supported by a Grant-in-Aid for Challenging Exploratory Research (No. 22650116) and the Innovative Techno-Hub for Integrated Medical Bio-imaging of the Project for Developing Innovation Systems, from the Ministry of Education, Culture, Sports, Science and Technology (MEXT), Japan.

\section{REFERENCES}

[1] J.C. Allred, R.N. Lyman, T.W. Kornack, and M.V. Romalis, "HighSensitivity Atomic Magnetometer Unaffected by Spin-Exchange Relaxation," Phys. Rev. Lett., vol. 89, no. 13, p. 130801, 2002.

[2] I.K. Kominis, T.W. Kornack, J.C. Allred, and M.V. Romalis, "A subfemtotesla multichannel atomic magnetometer," Nature, vol. 422, pp. 596-599, 2003.

[3] D. Budker and M.V. Romalis, "Optical magnetometry," Nature Phys., vol. 3, pp. 227-234, 2007.

[4] S. Taue, Y. Sugihara, T. Kobayashi, S. Ichihara, K. Ishikawa, and N. Mizutani, "Development of a Highly Sensitive Optically Pumped Atomic Magnetometer for Biomagnetic Field Measurements: A Phantom Study," IEEE Trans. Magn., vol. 46, no. 9, pp. 3635-3638, 2011.

[5] S. Taue, Y. Sugihara, T. Kobayashi, K. Ishikawa, and K. Kamada, "Magnetic Field Mapping and Biaxial Vector Operation for Biomagnetic Applications Using High-Sensitivity Optically Pumped Atomic Magnetometers," Jpn. J. Appl. Phys., vol. 50, p. 116604, 2011.
[6] Y. Ito, H. Ohnishi, K. Kamada, and T. Kobayashi, "Sensitivity Improvement of Spin-Exchange Relaxation Free Atomic Magnetometers by Hybrid Optical Pumping of Potassium and Rubidium," IEEE Trans. Magn., vol. 47, no. 10, pp. 3550-3553, 2011.

[7] M.V. Romalis, "Hybrid Optical Pumping of Optically Dense AlkaliMetal Vapor without Quenching Gas," Phys. Rev. Lett., vol. 105, no. 24, p. 243001, 2010.

[8] A. Gusarov, D. Levron, E. Paperno, R. Shuker, and A. Ben-Amar Baranga, "Three-Dimensional Magnetic Field Measurements in a Single SERF Atomic-Magnetometer Cell”, IEEE Trans. Magn., vol. 45, no. 10, pp. 4478-4481, 2009. 